\title{
PROGRESSION IN HIGH ENTROPY ALLOYS DEVELOPMENT TOWARD MATERIALS CHEMISTRY APPLICATIONS
}

\author{
A.U. Samuel ${ }^{1, \bigotimes}$, O.S.I. Fayomi ${ }^{2}$, O.A. Omotosho ${ }^{1}$, F. Zulkifli ${ }^{3}$, W.B. Wan Nik ${ }^{3}$ \\ and K.M. Oluwasegun ${ }^{4}$ \\ ${ }^{1}$ Department of Mechanical Engineering, Covenant University, Ota, Ogun State, Nigeria. \\ ${ }^{2}$ Department of Mechanical and Biomedical Engineering, Bells University of Technology, \\ Ota Ogun State, Nigeria \\ ${ }^{3}$ Faculty of Ocean Engineering Technology and Informatics, Universiti Malaysia Terengganu, \\ 21030 Kuala Nerus, Terengganu, Malaysia \\ ${ }^{4}$ Department of Mechanical and Manufacturing Engineering, University of Manitoba, Winnipeg, \\ Manitoba, Canada \\ ${ }^{\square}$ Corresponding Author: ayubasushe@gmail.com
}

\begin{abstract}
High entropy alloys (HIETAs) are currently of immense research importance in sciences and material engineering. Unlike the traditional alloys, which often contain two base components, HIETAs contain many fundamental elements. These essential elements are regarded as high in quantity compared to the traditional alloys. The advent of HIETAs necessitated researchers to start asking fundamental questions that face up to the theory, composition and techniques of preparation of the conventional alloys. In this paper, the review of the most recent studies on vital subject matters associated with HIETAs was presented. In addition, the uniqueness in the behavior of HIETAs was also examined. These include high corrosion and wear resistance, excellent mechanical performance at elevated temperature, exceptional rupture resistance, outstanding ductility, high cryogenic or extremely low-temperature strength, super-Para magnetism, and very high conductivity. The HIETAs are now perceived as potential materials for advance and novel applications, which necessitates further investigation due to their structures and richness of design.
\end{abstract}

Keywords: HIETAs, Alloys, Materials, Corrosion, Structures

RASĀYAN J. Chem., Special Issue, 2021

\section{INTRODUCTION}

The production of alloys has been through the traditional concept, which employs base constituents. ${ }^{1,2}$ As human beings advance, their ability to invent materials such as alloys has progressed from simple to multifaceted compositions. This has consequently improved functions and performances and promotes the advancements of human civilization. Various alloying elements have been employed as reinforcement to enhance engineering materials' electrochemical, thermal, mechanical, and electrical properties. However, the paradigm has shifted to high entropy alloys (HIETAs) in recent times due to their intrinsic microstructural characteristics and other remarkable properties, such as high strength, exceptional corrosion resistance, and high resistance to fracture and thermal stability. As a result of these properties, HIETAs overwhelm the traditional alloys with several potential applications. ${ }^{3}$ The HEATs are being used for barely a decade and have stimulated new ideas and encouraged the investigation of the enormous composition space presented by multi-principal element alloys (MPEAs). ${ }^{4}$ Researchers have shown that HIETAs have vast potential in material development. They are metallic alloy systems that comprise near equiatomic concentrations of four or more alloying elements ranging from 5 to 35 at.\%. ${ }^{5,6}$

The development of HEATs with properties that can substitute the conventional alloys used to manufacture advanced engineering components has been envisaged by researchers due to common defects, reduced cost, and high efficiency of operation. The HEATs have been produced through a variety of manufacturing techniques in recent years. ${ }^{7}$ Some of the HEATs development methods are; arc melting, 
Bridgman solidification, mechanical alloying, sputtering, thermal spray, induction melting, laser cladding, electrodeposition, spark plasma sintering and physical vapor deposition. ${ }^{8,9}$ The HEATs as a new class of materials have several existing potential applications, including submarines, state-of-the-art race cars, spacecraft, jet aircraft, nuclear reactors, long-range hypersonic missiles and nuclear weapons. ${ }^{10}$

Furthermore, studies have established that several HEATs exhibit a great strength-to-weight ratio, with a special measure of tensile strength, fracture resistance, and oxidation and corrosion resistance than the usual. ${ }^{11}$ The mechanical properties of any HEATs are a function of their crystal structure. Body-centered cubic HEAs characteristically have low ductility and high yield strength, whereas face-centered cubic HEATs possess high ductility and low yield strength. Overwhelmingly, HEAs' high mixing entropy effect promotes the formation of chaotic or disordered solid solutions of hexagonal closed-pack (HCP), FCC or BCC arrangement, as a replacement for complex intermetallic compounds. ${ }^{12}$ High entropy alloys like $\mathrm{CoCrFeMnNi}$ exhibited excellent mechanical properties and high fracture resistance at low temperature, increasing yield strength and ductility at a reduced temperature, from room to $350{ }^{\circ} \mathrm{C}$. This was accredited to the origination of nanoscale twin boundary, a supplementary mechanism of deformation that was not present at elevated temperatures. It may be found helpful for low-temperature structural material applications due to high toughness or energy absorbing material applications. ${ }^{13}$ However, successive research has shown that alloys of lower entropy with more minor elements or non-equiatomic compositions could have higher toughness or strength. ${ }^{14}$ Higher endurance and fatigue limit compared to conventional titanium alloys and steel was observed with $\mathrm{A} 10.5 \mathrm{CoCrCuFeNi} \mathrm{HEA}$, while samples of $\mathrm{NbTaMoW}$ exhibit astonishingly high yield strengths between 4 and $10 \mathrm{GPa}$, worthwhile ductility and high thermal stability, thus termed a material for potentially high temperature and stress application. ${ }^{15} \mathrm{In}$ this paper, the properties of HIETAs were studied. Superior structural applications of the alloys were also emphasized.

\section{Theory of HIETAs}

\section{EXPERIMENTAL}

High entropy alloys are generally known to contain at least five key elements, while the low and medium entropy alloys have one or two and two to four principal elements, respectively. Chemical composition increase with an increase in principal elements. This implies that HIETAs exhibit larger chemical composition compared to others. Understanding the possible interactions between the phases and mixing entropy is a vital subject of concern for manufacturers in their production. Due to this concern, researchers have asked many questions on the entropy of HIETAs. Consequently, the mixing entropy

could be written as $\Delta \mathrm{Smix}=-\mathrm{Kg} \sum_{\mathrm{i}=1}^{\mathrm{E}} \mathrm{M} i \mathrm{ln} \mathrm{Mi}$. Where $\mathrm{Kg}=$ the gas constant, $\mathbf{M i}=$ the ith element's molar fraction and $\hat{E}=$ the amount of metals contained in the mix. ${ }^{16-18}$

Researchers have also shown that phases of HIETAs usually seem to have tremendously smaller than Gibb's rule would allow, it was anticipated that configurational entropy offers a chief stabilizing effect in alloys of several phases. Also, many studies could not authenticate phases available in HIETAs; thus, it becomes essential to cautiously come up with the stability before recognizing the number of phases. Though substantiating the total thermo-dynamic stability of alloys was regarded as impossible via experimental means nevertheless, in the estimation of the stability of HIETAs, there are significant characteristics the research ought to have. Appropriate heat treatments ought to be selected for the homogeneity of the cast alloy and encouragement of decomposition of phases. Near-stable microstructures are produced by processing the cast HIETAs and the required confirmation on their stabilities can be made through careful characterization. Most studies have shown that many HIETAs are BCC, FCC, or a mixture of both because of their high configurational incorporation entropy. These HIETAs are organized configurationally to accomplish potential purposes including, high wear and corrosion resistance, improved ductility and hardness, etc..$^{19,20}$

\section{Methods of Fabrication of HIETAs}

High entropy alloys are complex to produce using the existing methods a few years back, and characteristically call for costly materials and specialty processing procedures. The HIETAs are predominantly manufactured using techniques that depend on the phase of metals - provided the metals 
combined while in a gaseous, solid or liquid state. In recent times, most high entropy alloys have been manufactured through liquid-phase techniques such as Bridgman solidification, induction melting and arc melting. Generally, solid-state processing is carried out by mechanical alloying employing a ball mill of high energy. This technique generates powders that can further be processed by the traditional powder metallurgy techniques or spark plasma sintering. This technique allows for the production of alloys not easy or unfeasible to manufacture using casting. Gas-phase processing involves molecular beam epitaxy (MBE) or sputtering that can be utilized carefully to control various elemental compositions to obtain high-entropy metallic films. ${ }^{21}$

The HIETAs have also been produced using other techniques such as electrodeposition, laser cladding and thermal spray. In the manufacturing of HIETAs employing mechanical alloying, the essential constituents are milled. Two phases known as terminal phases are often contained in the product. These phases are the amorphous and solid solution phases. To avoid welding or bonding of powder, the process of milling can first be done in the presence of liquid nitrogen. Several alloys are produced from cold casting, but instead of cast and melting, mechanical alloying is utilized for alloys preparation where constituent elements exhibit high vapour pressure. ${ }^{22,23}$

\section{Uniqueness and Dynamism of HIETAs}

Several authors have examined the characteristics of these alloys. There are indications that fundamental concerns such as the relationship between the mixing of the alloys constituent and the heating effect have not been entirely resolved. Nevertheless, HIETAs are said to have superior mechanical properties, outstanding physical characteristics, and super-high rupture toughness superior to many alloys. Their outstanding strengths are only comparable to some structural ceramics. They also possess exceptional resistance to corrosion and high. ${ }^{24}$ The remarkable properties of HIETAs have mainly been attributed to:

i. The effect of kinetic sluggish diffusion slows down the growth of the nucleus, thus aiding the formation of the precipitates (nano size).

ii. The severe distortion consequence of the structure lattice provides extra vigor and thus assists the kinetics in the alloys.

iii. The behavior of the cocktail, which could lead to enhancement in properties.

iv. The high entropy effects, which could stabilize the thermodynamics of the solutions in a single phase.

These descriptions explain the complexities awaiting clarifications and essential procedures for examining the configuration and behaviors association in HIETAs. ${ }^{25}$

Exceptional Ductility and Fracture Toughness of HIETAs at Cryogenic and Ambient Temperatures Reduction in ductility has been the result of the increase in strength in nearly all traditional alloys, and in most cases, the outcome of the decline in strength has been an enhancement in ductility. Nevertheless, several studies have attempted to develop alloys that possess high strength and reasonable ductility through the refinement of grains and grain boundaries of the alloys. This has also been achieved via the use of nano-twins for the development of microstructure gradient. Stacking faults (SFs) are predominant in most alloys. The energy of these SFs is essential in the development of twinning. By and large, the higher the energy of the SF, the more complex it is for the development of twinning. It is worth mentioning that many HIETAs exhibit low SF energy, which could even be immensely lower compared to some alloys of copper and aluminum, and steel. ${ }^{26}$

Stacking faults (SFs) with low energy enhances the splitting up of dislocation into partial and thus restrain the climbs and cross-slip dislocation. Due to low SFs energy, enhancement in yield strength (YS) can be attained for alloys. This behavior could lead to the production of twinning-induced plasticity, therefore resulting in enhanced ductility. Recently, several HIETAs have been observed to exhibit FCC with ample SF energy and single-phase possesses no small-sized twinning when deformed at ambient temperature. More so, some low SF energy single-phase HIETAs exhibit high ductility, in contrast with the traditional alloys. Fascinatingly, nano-twinning could perhaps not be the lone strengthening technique of alloys. Another method, such as solid solution hardening, has played an essential function in alloys fortification. ${ }^{27}$ 
The Single-phase FCC (SPF) HIETAs have attracted the attention of cryogenic applications due to their exceptional physical and mechanical properties such as high fracture toughness, excellent ductility and outstanding strength. The SPF HIETAs also reportedly exhibit exceptional scratch resistance with high strengths similar to or beyond those of the greatly revered cryogenic steels like the austenitic stainless steels and nickel steels. According to some researchers, the arrival of nano-twinning was the major root for the improvement in mechanical properties at cryogenic and room temperatures. ${ }^{28}$ Nonetheless, the relatively low YSs of the SPF HIETAs make them inadequately strong for some vital applications. Enthrallingly, $\gamma^{\prime}$ (gamma prime) reinforced HIETAs can exhibit improved YSs better than or equivalent to $1 \mathrm{GPa}$ at cryogenic and room temperatures. Similar to the SPF HIETAs, improvement in strength and ductility may be noticed at a deformation temperature of approximately $77 \mathrm{~K}$, showing exceptionally high ultimate tensile strength, enhanced ductility and improved yield strength of nearly $50 \%$. Such exceptional characteristics were ascribed to the nano gap SF. The outstanding strain-hardening prospect, high yield, and the extraordinary ductility of HIETAs with $\gamma^{\prime}$ reinforced phases make them cryogenically and hugely required. ${ }^{29}$

\section{The Outstanding Mechanical Characteristics of HIETAs}

As declared from the onset, 'the substantial mixing of elements in HIETAs enhances the growth of the solid solutions of single-phase. ' This, accordingly, makes the production of alloys for easy. The outstanding property of HIETA is accomplished at high temperatures due to the sluggish diffusion or circulation of the constituents that make up the mixture. Most refractory HIETAs high-temperature singlephase BCC structures possess high YS comparable to those of traditional superalloys. The strength of the refractory HIETAs is similar to that of Inconel 718 (In 718) superalloy at low temperatures. Nevertheless, In 718 significantly becomes softer thermodynamically above $600{ }^{\circ} \mathrm{C}$, while the HIETAs still uphold their dynamism at temperatures above $1200{ }^{\circ} \mathrm{C} .{ }^{30}$

Also, the recent of most researchers on the advancement of HIETAs has substantiated that, in addition to the remarkably high-temperature application potentials of the refractory HIETAs, their densities could be significantly minimized by substituting the heavier elements contained in compositional design with lighter constituents. Heavier elements such as Tantalum (Ta) and Tungsten (W) in compositional design could be substituted with lighter ones such Tin (Sn), Aluminium (Al), Zinc (Zn), Titanium (Ti) and Chromium (Cr) to reduce the weight of the end product. For some applications, while the formulation and production of HIETAs have been affected by these alloying elements, the YS at room and high temperatures were improved. ${ }^{31}$ For advanced applications such as gas turbines and some aerospace components where high temperatures are needed, one of the foremost mechanical characteristics required is a high creep resistance (CR). The $\gamma^{\prime}$ reinforced HIETAs have been reported to possess great CR due to the reasonable thermal stability of precipitate of $\gamma^{\prime}$, their low SF energy and enormous creep activation energy. Also reported is that the $\gamma^{\prime}$ reinforced HIETAs take a long time to rapture relative to several superalloys under similar operational circumstances. ${ }^{32}$

Asides from the outstanding mechanical characteristics of most HIETAs, the majority also exhibit attractive and distinctive serviceable properties due to the high entropy of composition. This attribute limits precipitation to nano-meter scale or causes no precipitation at all. Many HIETAs have been revealed to have high paramagnetic characteristics, ${ }^{33}$ a few others possess high conducting ability at significant at extremely high and low temperatures. ${ }^{34}$ These properties are an indication that HIETAs can be used for electromagnetic applications such as magnetic resonance imaging and particle accelerators.

\section{Incredible Hardness and Strength of HIETAs}

Alloys produced for engineering applications are required to possess high hardness, outstanding strength and low density. These properties facilitate the employment of some of these alloys for aircraft, a component of automobiles, marine, etc, where material density rule is vital for achieving the required outfitted energy. As a result of this requirement, engineers have manufactured HIETAs with lightweight and outstanding strength. $\mathrm{Al}_{20} \mathrm{Be}_{20} \mathrm{Fe}_{10} \mathrm{Si}_{15} \mathrm{Ti}_{35}$, a HIETA material, was produced blending low-density elements and is observed to possess incredible mechanical performance, superior to most traditional and 
superalloys. The hardness and strength of these alloys were attributed to the presence of cluttered BCC solutions. Similarly, many HIETAs possess drastically high compressive YS at ambient temperature. ${ }^{35}$ In recent times HIETAs with extremely low densities, ultra-high-strength and hexagonal close-packed (HCP) compositions are being produced using mechanical alloying routes. Studies revealed that these alloys are extensively harder compared to traditional alloys. Though their specific strengths are several times greater relative to the traditional low-weight elements like $\mathrm{Ti}, \mathrm{Zn}$ and $\mathrm{Cu}$ but analogous to some ceramic carbide. ${ }^{36}$

\section{Oxidation Protection Properties of HIETAs}

Inclusions of elements such as $\mathrm{Al}, \mathrm{Cr}, \mathrm{Zn}, \mathrm{Zr}$ and $\mathrm{Ti}$ have appreciably enhanced materials resistance to oxidation. Even though the oxidation characteristics of $\gamma^{\prime}$ reinforced HIETAs are sparse in literature, the isothermal oxidation of $\gamma^{\prime}$ reinforced HIETAs at high temperature shows no presence of oxide spallation. Many diverse solutes in $\gamma^{\prime}$ reinforced HIETAs have resulted in the production of complexes in oxide form. HIETAs are reported to possess critical structures. These oxidations structures are the external layer, the internal layer (made up of a complex mixture of some compounds), and the layer beneath (usually consists of oxides such as $\mathrm{Al}_{2} \mathrm{O}_{3}$ and $\mathrm{Cr}_{2} \mathrm{O}_{3}$, opposing internal oxidation particularly in HIETAs whose compositional formulation contains $\mathrm{Cr}$ and $\mathrm{Al}$. It has been established that $\mathrm{Al}_{2} \mathrm{O}_{3}$ offers better surface protection at high temperatures relative to $\mathrm{Cr}_{2} \mathrm{O}_{3}$. This is a result of the lower permeability of oxygen and higher thermal stability of $\mathrm{Al}_{2} \mathrm{O}_{3}$. Beyond $950{ }^{\circ} \mathrm{C}, \mathrm{Cr}_{2} \mathrm{O}_{3}$ often give up its stability and could slowly transform into an unstable $\mathrm{CrO}_{3}$. Fundamentally, the exceptional oxidation resistance of $\gamma^{\prime}$ reinforced HIETAs at high temperatures is due to the development of $\mathrm{Al}_{2} \mathrm{O}_{3}$ film, which shields the alloys from hot oxidation and protects the material's characteristics for structural and advanced functional efficiency. ${ }^{37,38}$

\section{RESULTS AND DISCUSSION \\ Current and Prospective Applications of HIETAs}

The most recently produced HIETAs have been employed to manufacture materials in automobiles, aerospace, power plants and marines industries. Several high-temperature applications have required the use of HIETAs. For instance, in the production of compressor blades and turbine fans of aircraft's engines, HIETAs have been the potential replacement for materials such as steel and titanium alloys because high reliability, higher efficiency and highly high operational fatigue are required. In aircrafts supersonic in nature, the heat generated from friction increases the exterior temperature beyond what the objects can withstand. However, with HIETAs the heat is either absorbed or suppressed due to high creep resistance and high fatigue properties. They have also been employed for the fortification of tools exteriors via coating due to their high corrosion and wear resistance and high CR. The HIETAs can be coated on the top of tools in powdered form and rod. They are also sprayed thermally to offer complex exterior technology, which involves a thick coating of wear and corrosion inhibition devices on tools, nozzles, moulds and dies for their protection. ${ }^{39}$

Furthermore, HIETAs have been employed as binders. These alloys can potentially substitute the regular ones after passing have been sintered. HIETAs can be used without grain refiner. In micro-joining, HIETAs can be used as fillers since the conventional brazing method leads to breakage during the joining of metal and ceramic devices. This is because of the presence of numerous boundary complexes and residual stress. The complexes could be overcome by balancing the ratio of strength and ductility of the HIETAs and the effect of the solid solution phase of the alloys. These HIETAs have been deposited on cooking materials to improve their wear and electrochemical properties. The shallow temperature characteristics of most HIETAs can also explore for applications in liquid gas compartments since their mechanical can still be retained at extremely high temperatures. ${ }^{40}$

\section{CONCLUSION}

In this paper, the properties of HIETAs were studied, and the projections for superior structural applications were highlighted. HEATs exhibit a tremendous strength-to-weight ratio, with a particular measure of tensile strength, fracture resistance, as well as oxidation and corrosion resistance than the usual alloys. High entropy alloys are generally known to contain at least five critical elements, while the 
RASĀYAN J. Chem.

1-7| Special Issue | 2021

low and medium entropy alloys have one or two and two to four principal elements, respectively. In recent times, most high entropy alloys have been manufactured through liquid-phase techniques such as Bridgman solidification, induction melting and arc melting. Generally, solid-state processing is carried out by mechanical alloying employing a ball mill of high energy. The outstanding hardening prospect, high yield, and the extraordinary ductility of HIETAs with $\gamma^{\prime}$ reinforced phases make them cryogenically beautiful materials. The $\gamma^{\prime}$ reinforced HIETAs have been reported to possess excellent CR due to the thermal stability of $\gamma^{\prime}$ precipitate, their low SF energy and enormous creep activation energy. In producing compressor blades and turbine fans of aircraft's engines, HIETAs have been the potential replacement for materials such as steel and titanium alloys because these applications require high reliability.

\section{ACKNOWLEDGEMENT}

The authors would like to acknowledge the research support from Covenant University and Bells University of Technology to create a conducive environment for collaborative Interdisciplinary Research. The financial support from Universiti Malaysia Terengganu (Corrosion UMT-MG 1+3) and University of Manitoba, Winnipeg, Manitoba, Canada, is deeply appreciated.

\section{REFERENCES}

1. S. Gorsse, J. P. Couzinie and D. B. Miracle, Comptes Rendus Physique, 19(8), 721(2018), https://doi.org/10.1016/j.crhy.2018.09.004

2. D. Schaming and H. Remita, Foundations of Chemistry, 17(3), 187(2015), https://doi.org/10.1007/s10698-015-9235-y

3. W. Zhang, P. K. Liaw and Y. Zhang, Science China Materials, 61(1), 2(2018), https://doi.org/ https://doi.org/10.1007/s40843-017-9195-8

4. D. B. Miracle, and O. N. Senkov, Acta Materialia, 122, 448(2017), https://doi.org/10.1016/j.actamat.2016.08.081

5. H. J. Park, Y. S. Na, S. H. Hong, J. T. Kim, Y. S. Kim, K. R. Lim,... and K. B. Kim, Metals and Materials International, 22(4), 551(2016), https://doi.org/10.1007/s12540.016-6034-5

6. A. Takeuchi, K. Amiya, and K. Yubuta, Materials Chemistry and Physics, 210, 245(2018), https://doi.org/10.1016/j.matchemphys.2017.10.057

7. H. Dobbelstein, E. L. Gurevich, E. P. George, A. Ostendorf and G. Laplanche, Additive Manufacturing, 25, 252(2019), https://doi.org/10.1016/j.addma.2018.10.042

8. J. Cieslak, J. Tobola, K. Berent and M. Marciszko, Journal of Alloys and Compounds, 740, 264 (2018), https://doi.org/10.1016/j.jallcom.2017.12.333

9. W. Ji, W. Wang, H. Wang, J. Zhang, Y. Wang, F. Zhang and Z. Fu, Intermetallics, 56, 24(2015), https://doi.org/10.1016/j.intermet.2014.08.008

10.T. R. Prabhu, M. Arivarasu, Y. Chodancar, N. Arivazhagan, G. Sumanth, and R. K. Mishra, Tribology Letters, 67(3), 78(2019), https://doi.org/10.1007/s11249-019-1193-4

11.D. Yim, P. Sathiyamoorthi, S. J. Hong and H. S. Kim, Journal of Alloys and Compounds, 781, 89 (2019), https://doi.org/10.1016/j.jallcom.2018.12.119

12.M. Feuerbacher, M. Heidelmann and C. Thomas, Materials Research Letters, 3(1), 1(2015), https://doi.org/10.1080/21663831.2014.951493

13.F. Otto, A. Dlouh, C. Somsen, H. Bei, G. Eggeler and E. P. George, Acta Materialia, 61(15), 5743(2013), https://doi.org/10.1016/j.actamat.2013.06.018

14.A. J. Zaddach, R. O. Scattergood and C. C Koch, Materials Science and Engineering: A, 636, 373 (2015), https://doi.org/10.1016/j.msea.2015.03.109

15.Y. Zou, S. Maiti, W.Steurer and R. Spolenak, Acta Materialia, 65, 85(2014), https://doi.org/10.1016/j.actamat.2013.11.049

16.S. Guo, Materials Science and Technology, 31(10), 1223(2015), https://doi.org/10.1179/1743284715Y.0000000018

17.X. Fu, C. A. Schuh and E. A. Olivetti, Scripta Materialia, 138, 145(2017), https://doi.org/10.1016/j.scriptamat.2017.03.014 
RASĀYAN J. Chem.

1-7| Special Issue | 2021

18.Y. F., Ye, Q., Wang, J., Lu, C. T., Liu and Y. Yang, Materials Today, 19(6), 349(2016), https://doi.org/10.1016/j.mattod.2015.11.026

19.E. J. Pickering and N. G. Jones, International Materials Reviews, 61(3), 183(2016), https://doi.org/10.1080/09506608.2016.1180020

20.Z. S. Nong, Y. N., Lei, \& J. C. Zhu, Intermetallics, 101, 144(2018), https://doi.org/10.1016/j.intermet.2018.07.017

21.P. F.Yu, L. J. Zhang, H. Cheng, H. Zhang, M. Z. Ma, Y. C. Li and R. P. Liu, Intermetallics, 70, 82 (2016), https://doi.org/10.1016/j.intermet.2015.11.005

22.O. N. Senkov and D. B. Miracle, Journal of Alloys and Compounds, 658, 603(2016), https://doi.org/10.1016/j.jallcom.2015.10.279

23.A. Erdogan, T. Yener and S. Zeytin, Vacuum, 155, 64(2018), https://doi.org/10.1016/j.vacuum.2018.05.027

24.D. Li, and Y. Zhang, Intermetallics, 70, 24(2016), https://doi.org/10.1016/j.intermet.2015.11.002

25.Y. Zhang, T. T. Zuo, Z. Tang, M. C. Gao, K. A. Dahmen, P. K. Liaw and Z. P. Lu, Progress in Materials Science, 61, 1(2014), https://doi.org/10.1016/j.pmatsci.2013.10.001

26.P. Yu, R. Feng, J. Du, S. Shinzato, J. P. Chou, B. Chen... and A. Hu, Acta Materialia, 181, 491(2019), https://doi.org/10.1016/j.actamat.2019.10.012

27.Z. Wu, C. M. Parish and H. Bei, Journal of Alloys and Compounds, 647, 815(2015), https://doi.org/10.1016/j.jallcom.2015.05.224

28.M. Bonisch, Y. $\mathrm{Wu}$ and H. Sehitoglu, Scientific Reports, 8(1), 1(2018), https://doi.org/10.1038/s41598-018-28784-1

29. Y. Wang, B. Liu, K. Yan, M. Wang, S. Kabra, Y. L. Chiu and B. Cai, Acta Materialia, 154, 79(2018), https://doi.org/10.1016/j.actamat.2018.05.013

30.B. Gludovatz, E. P. George and R. O. Ritchie, The Journal of the Minerals, Metals, and Materials Society, 67(10), 2262(2015), https://doi.org/10.1007/s11837-015-1589-Z

31.J. Chen, X. Zhou, W. Wang, B. Liu, Y. Lv, W. Yang and Y. Liu, Journal of Alloys and Compounds, 760, 15(2018), https://doi.org/10.1016/j.jallcom.2018.05.067

32.T. K. Tsao, A. C. Yeh, C. M. Kuo, K. Kakehi, H. Murakami, J. W. Yeh and S. R. Jian, Scientific Reports, 7(1), 1(2017), https://doi.org/10.1038/s41598-017-13026-7

33.R. Kulkarni, B. S. Murty and V. Srinivas, 746, 194(2018), https://doi.org/10.1016/j.jallcom.2018.02.275

34.M. C. Gao, D. B. Miracle, D. Maurice, X. Yan, Y. Zhang and J. A. Hawk, Journal of Materials Research, 33(19), 3138(2018), https://doi.org/10.1557/jmr.2018.323

35.K. Tseng, Y. Yang, C. Juan, T. Chin, C. Tsai and J. Yeh, Science China Technological Sciences, 61(2), 184(2018), https://doi.org/10.1007/s11431-017-9073-0

36.C. E. Slone, J. Miao, E. P. George and M. J. Mills, Acta Materialia, 165, 496(2019), https://doi.org/10.1016/j.actamat.2018.12.015

37.H. M. Daoud, A. M. Manzoni, R. Volkl, N. Wanderka and U. Glatzel, Advanced Engineering Materials, 17(8), 1134(2015), https://doi.org/10.1002/adem.201500179

38.T. K. Tsao, A. C. Yeh, C. M. Kuo and H. Murakami, Entropy, 18(2), 1(2016), https://doi.org/10.3390/e18020062

39.J. W. Yeh and S. J. Lin, Journal of Materials Research,33(19), 3129(2018), https://doi.org/10.1557/jmr.2018.283

40.H. Zhang, Y. Pan, Y. Z. He, J. L. Wu, T. M. Yue and S. Guo, The Journal of the Minerals, Metals, and Materials Society, 66(10), 2057(2014), https://doi.org/10.1007/s11837-014-1036-6

[RJC-6413/2021] 